Technological University Dublin

ARROW@TU Dublin

2011-9

\title{
Testing, Modelling and Validation of Numerical Model Capable of Predicting Stress Fields Throughout Polyurethane Foam
}

Conor Briody

Technological University Dublin, conor.briody@tudublin.ie

Barry Duignan

Technological University Dublin, Barry.Duignan@tudublin.ie

Stephen Jerrams

Technological University Dublin, stephen.jerrams@tudublin.ie

Follow this and additional works at: https://arrow.tudublin.ie/cercon

Part of the Computational Engineering Commons, and the Polymer and Organic Materials Commons

\section{Recommended Citation}

Briody, C., Duignan, B. \& Jerrams, S. (2011) Testing, modelling and validation of numerical model capable of predicting stress fields throughout polyurethane foam. Proceedings of the 7th European Conference on Constitutive models for Rubber (ECCMR). Dublin, Ireland, 20 - 23 September, 2011.

This Conference Paper is brought to you for free and open access by the Centre for Elastomer Research at ARROW@TU Dublin. It has been accepted for inclusion in Conference Papers by an authorized administrator of ARROW@TU Dublin. For more information, please contact arrow.admin@tudublin.ie, aisling.coyne@tudublin.ie,gerard.connolly@tudublin.ie. Funder: ABBEST 


\title{
Testing, modelling and validation of numerical model capable of predicting stress fields throughout polyurethane foam
}

\author{
C. Briody, B. Duignan \& S. Jerrams \\ Dublin Institute of Technology, Bolton St, Dublin 1, Ireland
}

\begin{abstract}
Wheelchair seating systems are specialised for a number of reasons as users can have impaired mobility, which increases the possibility of pressure build up. These areas of high pressure frequently occur in the trunk region under the bony prominences known as the Ischial Tuberosities (IT), pressure ulcers may occur consequently. Polyurethane foam has been in use for some time in wheelchair seating systems as it exhibits good pressure relieving capabilities in most cases. However, little quantitative research has gone into foamed polymers, in comparison with conventional elastomeric materials. This lack of knowledge can ultimately lead to more time being spent in fitting, increased possibility of refitting and potentially an increase in trunk region pressures leading to the development of ulcers. Test results were used to accurately validate a Visco-Hyperfoam material model. Accurately simulating an indentation procedure using FE software verified the validation of the material model.
\end{abstract}

\section{INRODUCTION}

A pressure ulcer can be defined as a localised injury to the skin or underlying tissue, usually over a bony prominence, as a result of pressure, or pressure in combination with shear forces or friction (Black 2007). Pressure ulcers have the potential to diminish physical, psychological and social wellbeing and cause serious pain and discomfort which drastically decreases quality of life (Medicine 2001; Voss et al. 2005). There are two types of pressure ulcers, superficial and Deep Tissue Injury (DTI). Upon application of bodyweight, high pressure radiates outwards from bony prominences called the Ischial Tuberosities which are located on the pelvis. This high pressure can cause the damage termed DTI (Bouten, Oomens et al. 2003).Superficial ulcers occur on the outer layers of the skin tissue, although generally the extent of a superficial pressure sore is not as serious as a DTI. Improved understanding of the behaviour of the materials used in wheelchair seating can enable superior designs with improved pressure distribution. This will enhance comfort and support and potentially reduce the onset of pressure ulcers.

Polyurethane foam is an open celled elastomeric polymer and its constituent elastomer, polyurethane rubber, can undergo large and reversible deformations. Foamed polymeric material is known to exhibit three regions of different stress-strain behaviour in simple uniaxial compression: (i) approximately linear behaviour for strains less than about 0.05 ; this linear elasticity arises from the bending of the cell edges, (ii) a plateau region in which strain increases at constant or nearly constant stress up to a strain of roughly 0.6 ; this plateau arises from elastic buckling of the cell edges and (iii) a densification of the collapsed cell edges causing the foam to act as would its elastomeric constituent material. In this final region, known as the densification region, the slope of the stress-strain curve increases exponentially with strain as the crushed foam's cell struts and vertices come into contact (Gibson 1997). When the material reaches this level of compression, it is clinically referred to as 'bottomed out'.

Viscoelastic materials can be idealised as an intermediate combination of elastic solids and viscous liquids (Ward 2004). All polyurethane foams exhibit some degree of viscoelasticity (Mills 2007). Viscoelastic polyurethane foam is widely used in clinical seating as it offers excellent comfort and support due to its particular polymeric properties which are dependent on time, temperature and strain rate. The work presented in this paper is part of an ongoing investigation into improvements in the methodologies in the specification of viscoelastic polyurethane foams in wheelchair seating.

\section{MATERIAL TESTING}

\subsection{Materials tested}

Open-celled polyurethane seating foam with a density of $40 \mathrm{~kg} / \mathrm{m}^{3}$ was tested in this piece of work. Several empirical tests were conducted on foam samples and the results were used to represent the behaviour of the material using prediction based numerical material models, which would later be used for seating design optimisation.

\subsection{Uni-axial compression testing}

Uni-axial compression testing was conducted on the selected material in accordance with 'ISO 3386: Polymeric materials, cellular flexible - Determination of stress-strain characteristic in compression'. The compression tests were performed on a Lloyd LR $30 \mathrm{~K}$ materials testing machine which incorporated a calibrated $3 \mathrm{kN}$ Lloyd instruments load cell as shown in Figure 1. The test-piece was inserted centrally between two horizontal platens in the testing machine. For the first test, the sample was compressed by 
$70 \%$ of its initial height at a strain rate of $5 \mathrm{~mm} / \mathrm{min}$. This continuous cycle was repeated immediately three times and on the fourth compression cycle, load-deflection data was recorded. The initial 3 cycles applied to the virgin foam sample removed most of the Mullins effect (Mullins 1969). After recording the load-deflection data, the sample was decompressed. This test procedure was then repeated at strain rates of 50,100, 250 and $500 \mathrm{~mm} / \mathrm{min}$.

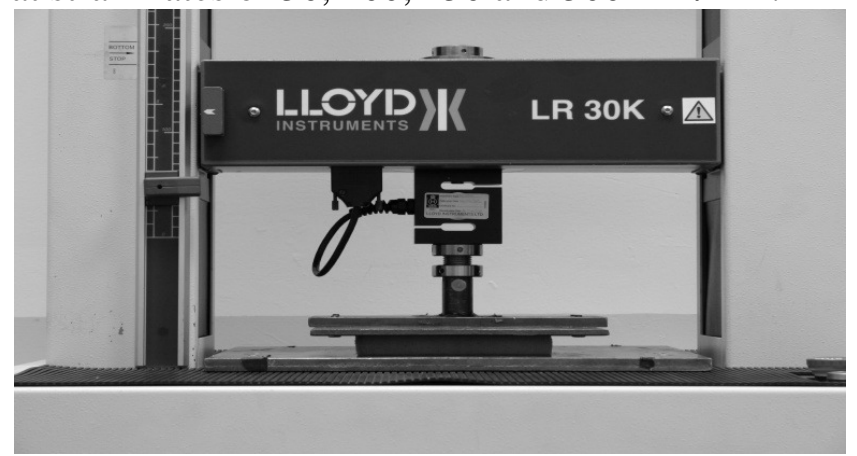

Fig.1: Uni-axial compression testing set-up

\subsection{Constant displacement stress relaxation testing}

The same test set-up as described in section 2.2 was used to conduct constant displacement stress relaxation testing following the guidelines proposed in 'ISO 3384 - Rubber, vulcanized or thermoplastic Determination of stress relaxation in compression Part 1: Testing at constant temperature'. This test procedure measured the decrease in counterforce exerted by a test piece of polymer foam which was compressed to a constant deformation. Samples were compressed at a strain rate of $250 \mathrm{~mm} / \mathrm{min}$ and held at $80 \%$ compressive strain for extended time periods of up to 8 hours. This time was chosen to replicate a typical daily occupancy of a wheelchair user. The dissipating force was monitored over the entire period of the test.

\subsection{Simple shear testing}

Shear (rigidity) modulus testing was conducted in accordance with 'ISO 1827: Rubber, vulcanized or thermoplastic - Determination of modulus in shear'. Samples were bonded with cyanoacrylate adhesive on both sides to the rigid plates during testing. The shear load was applied at a rate of $4 \mathrm{~mm} / \mathrm{min}$ until sample failure. A minority of the shear tests failed at relatively low strain values due to adhesive failure any test that failed at less than $100 \%$ shear strain was regarded as unrepresentative of material behaviour and disregarded.

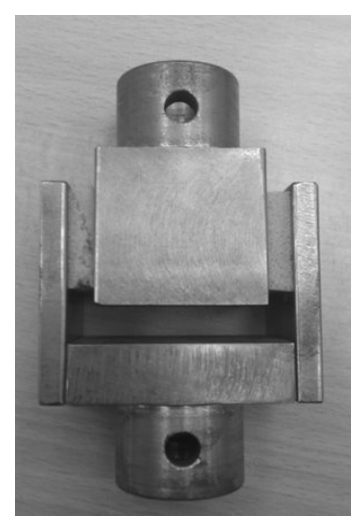

Fig.2: Shear test set-up, sample on left failed due to shear

\subsection{Indentation Force Deflection testing}

Indentation Force Deflection (IFD) tests were conducted on the foam. A circular indenter based on 'ISO:2439, "Flexible Cellular Polymeric MaterialsDetermination of Hardness (Indentation Technique)' but scaled down to $81.2 \mathrm{~mm}$ in diameter, to be compatible with the $150 \mathrm{~mm}$ square test-pieces. This indenter was axially indented into the foam samples up to $65 \%$ of sample height using the Lloyd Instruments testing machine. The result of this test is presented in section 4.1 and compared to the results suggested by a Finite Element (FE) simulation.

\section{DEVELOPMENT OF MATERIAL MODEL FROM TEST DATA}

\subsection{Uni-axial test data fit}

Nominal uniaxial compression test data sets, obtained from the procedure described in section 2.2, were used to calculate the constants for the $2^{\text {nd }}$ order form of Ogden's Hyperfoam material model (Ogden 1972; Simulia 2010) described in Equation 1.

$$
\begin{aligned}
& U=\sum_{i=1}^{N} \frac{2 \mu_{i}}{\alpha_{i}^{2}} \cdot\left[{\widehat{\lambda_{1}}}^{\alpha_{i}}+{\widehat{\lambda_{2}}}^{\alpha_{i}}+{\widehat{\lambda_{3}}}^{\alpha_{i}}-3+\right. \\
& \left.\frac{1}{\beta_{i}} \cdot\left(\left(J^{e l}\right)^{-\alpha_{i} \beta_{i}}-1\right)\right]
\end{aligned}
$$

where $\mathrm{N}$ is the order of fitting, $\mu_{i}, \alpha_{i}$, and $\beta_{i}$ are temperature-dependent material parameters to be determined by curve-fitting test data to the model and $J^{e l}$ is given by:

$\hat{\lambda}_{i}=\left(J^{t h}\right)^{-\frac{1}{3}} \stackrel{\text { yields }}{\longrightarrow} \widehat{\lambda_{1}} \widehat{\lambda_{2}} \widehat{\lambda_{3}}=J^{e l}$

where $\lambda_{i}$ is the principal stretch ratio, $J_{e l}$ and $J_{t h}$ are the elastic and thermal volume ratios respectively and can be defined by the following equations $J_{e l}=\frac{J}{J_{t h}}$ and $J_{t h}=\left(1+\epsilon_{t h}\right)^{3} . J$ is the total volume ratio and the thermal strain, $\varepsilon_{t h}$, is calculated from 
the temperature and the isotropic thermal expansion coefficient.

The test data modelled here is taken from a uniaxial compression test conducted on a sample at a strain rate of $5 \mathrm{~mm} / \mathrm{min}$. It was assumed that Poisson's ratio $(v)=0$ and that the lateral principal stretches, $\lambda_{1}$ and $\lambda_{3}$ can be considered to be zero. Equation 3 is used to calculate the nominal engineering stress, $\sigma_{2}$, in the $\lambda_{2}$ direction.

$\sigma_{2}=\frac{\partial U}{\partial \lambda_{2}}=\frac{2}{\lambda_{2}} \sum_{i=1}^{N} \frac{\mu_{i}}{\alpha_{i}}\left[\lambda_{2}^{\alpha_{i}}-J_{e l}^{-\alpha_{i} \beta_{i}}\right]$

Abaqus automatically fits the parameters $\mu, \alpha$ and $\beta$ using a non-linear least squares optimisation procedure. The parameters used in the curve fit shown in figure 3 , are given in Table 1:

\begin{tabular}{|l|l|l|l|}
\hline $\mathbf{N}$ & $\boldsymbol{\mu ( P a )}$ & $\boldsymbol{\alpha}$ & $\boldsymbol{\beta}$ \\
\hline $\mathbf{1}$ & 44185.6 & 21.4556 & 0 \\
\hline $\mathbf{2}$ & 3.7050 & -6.8900 & 0 \\
\hline
\end{tabular}

Table 1: Coefficients of Ogden Hyperfoam model for uniaxial compression case

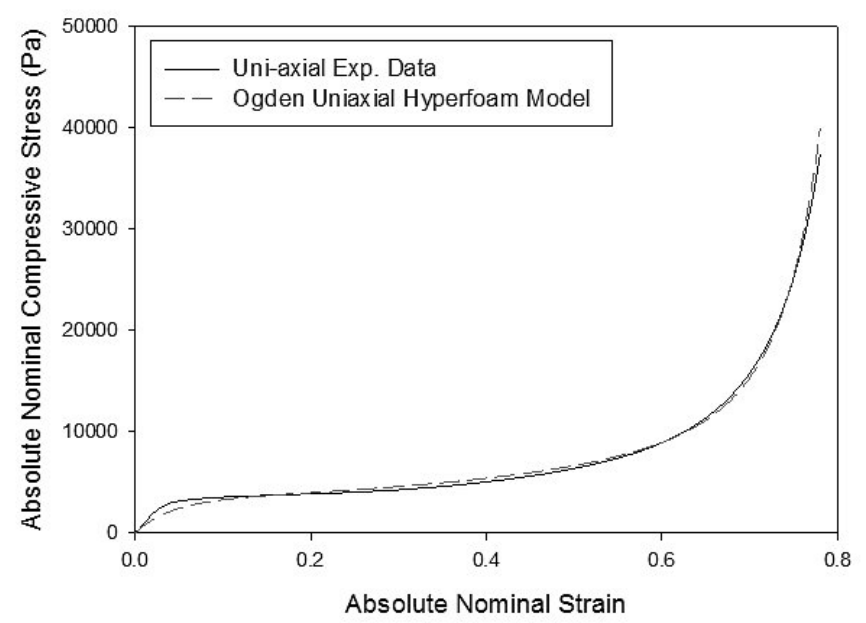

Fig.3: Uniaxial compression data for a sample compressed at a crosshead speed of $50 \mathrm{~mm} / \mathrm{min}$, to a compressive strain of $80 \%$ at $20^{\circ} \mathrm{C}$ compared with Ogden Hyperfoam Model curve-fit

Overall the model fits accurately to the experimental data in Figure 3. Some slight error is noticeable in the initial elastic region as the test data stiffer material than the model predicts. The shape of this initial elastic region is strongly dependent on the $\mu_{1}$ material constant. This error can be eradicated by weighting the data towards the lower values of strain; however this would introduce error in the higher strain range. Error at lower values of strain was deemed less important than error at the more critical higher strain values.

\subsection{Simple shear test data fit}

Simple shear test data, extracted from the procedure described in Section 2.3, was also curve-fitted to the material model as during service the material will deform in both compression and shear. Simple shear was fitted using Equation 4

$T_{S}=\frac{\partial U}{\partial \gamma}=\sum_{j=1}^{2}\left[\frac{2 \gamma}{2\left(\lambda_{j}^{2}-1\right)-\gamma^{2}} \sum_{i=1}^{N} \frac{\mu_{i}}{\alpha_{i}}\left(\lambda_{j}^{\alpha_{i}}-1\right)\right]$

where $\gamma$ is the shear strain and $\lambda_{j}$ are the two principal stretches in the plane of shearing and are related to the shear strain by

$\lambda_{1,2}=\sqrt{1+\frac{\gamma^{2}}{2} \pm \gamma \sqrt{1+\frac{\gamma^{2}}{4}}}$

Abaqus used the same curve-fit procedure to calculate representative parameters of the Ogden hyperfoam model to best fit the curve to the experimental simple shear data. The parameters calculated and shown in Table 2, give a very accurate curve fit for the shear loading mode as can be seen in Figure 4.

\begin{tabular}{|l|l|l|l|}
\hline $\mathbf{N}$ & $\boldsymbol{\mu}(\mathbf{P a})$ & $\boldsymbol{\alpha}$ & $\boldsymbol{\beta}$ \\
\hline $\mathbf{1}$ & 7242.15 & 5.99916 & 0 \\
\hline $\mathbf{2}$ & 7242.15 & -5.99916 & 0 \\
\hline
\end{tabular}

Table 2: Coefficients of Ogden Hyperfoam model for simple shear case

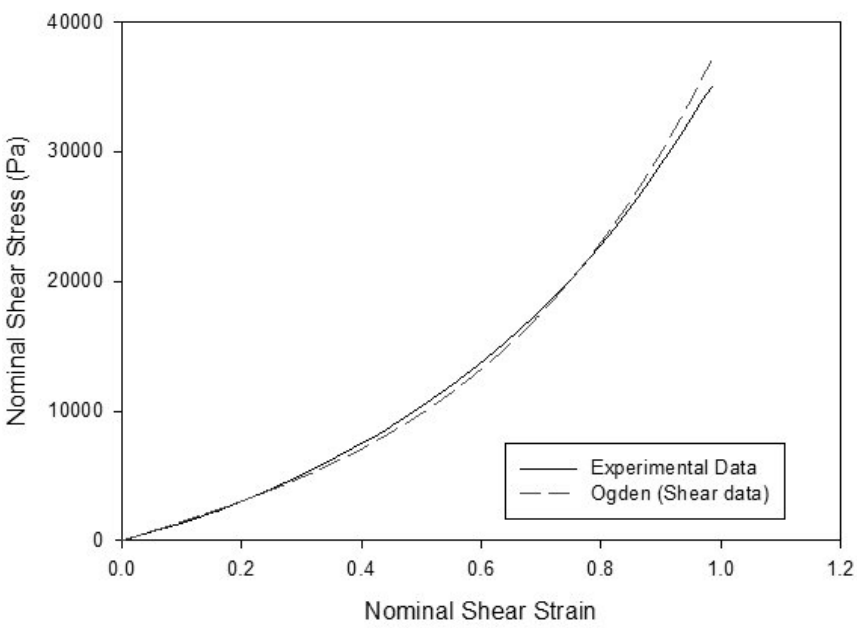

Fig. 4: Simple shear data for a sample sheared at a crosshead speed of $4 \mathrm{~mm} / \mathrm{min}$ at $20^{\circ} \mathrm{C}$ compared to Ogden Hyperfoam Model curve-fit.

\subsection{Combination of uniaxial compression data and simple shear data}

A compromise was made in the accuracy of the two curve fits, uniaxial compression and simple shear to ensure the model could predict stress fields under complex combinations of both modes of deformation. Data sets from both forms of deformation were used in the calculation of the Ogden hyperfoam constants. This meant that while some accuracy was lost when compared to the fits for each separate mode, the model was more robust. The derivation of model 
constants followed best practise guidelines (Simulia 2010), constants were derived from test modes which were the most relevant to the materials in-use mode of deformation. The accuracy of the model in simulating a uniaxial test is demonstrated graphically in Figure 5. Three different curve fits, uniaxial, shear and uniaxial plus shear, are plotted against the uniaxial compression test data curve in Figure 5. A compromise can clearly be seen in the accuracy of the curve-fit made in using both modes of deformation to derive material parameters when compared with using the uniaxial mode of deformation by itself. The inaccurate curve fit derived using simple shear test data demonstrates the importance of using more than one mode of deformation when simulating complex modes of deformation. The material parameters derived using both deformation mode data sets are shown in Table 3.

\begin{tabular}{|l|l|l|l|}
\hline $\mathbf{N}$ & $\boldsymbol{\mu ( P a )}$ & $\boldsymbol{\alpha}$ & $\boldsymbol{\beta}$ \\
\hline $\mathbf{1}$ & 12740.4000 & 7.2810 & 0 \\
\hline $\mathbf{2}$ & 2.7459 & -5.7311 & 0 \\
\hline
\end{tabular}

Table 3: Coefficients of Ogden Hyperfoam model for combination case

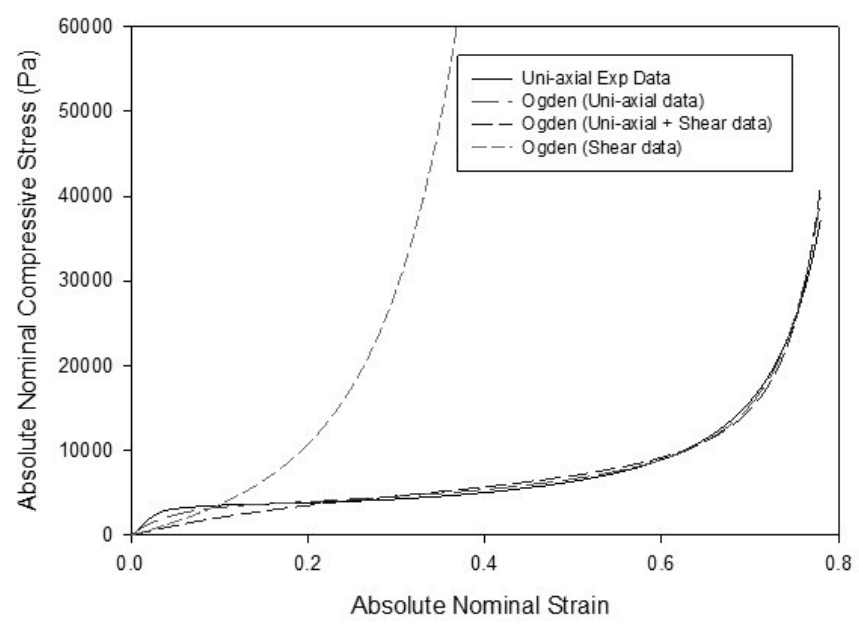

Fig.5: Uni-axial compression data for foam sample compressed at a crosshead speed of $50 \mathrm{~mm} / \mathrm{min}$ to a compressive strain of $80 \%$ at $20^{\circ} \mathrm{C}$ compared with an Ogden Hyperfoam Model curve-fit for the uniaxial, shear and combination cases.

The accuracy of the model when a shear test is undertaken was also studied. The material coefficients were determined with uniaxial data, shear data and a combination of both data sets. The accuracy of each of the curve fits is compared in Figure 6. Again the model parameters derived from the mode of deformation in question generate the most accurate model, as was the case for the uni-axial test data modelling.

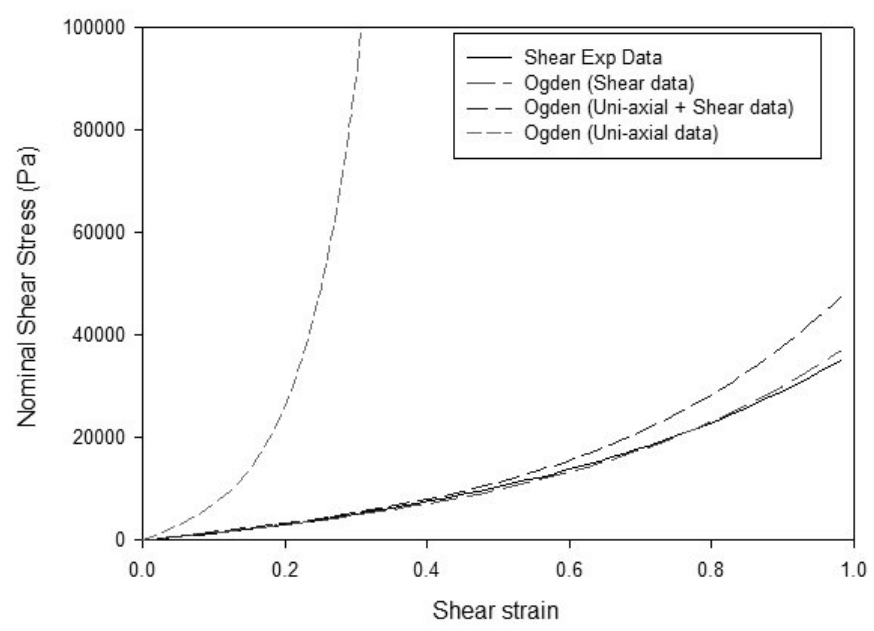

Fig.6: Simple shear data for a sample sheared at a crosshead speed of $4 \mathrm{~mm} / \mathrm{min}$, at $20^{\circ} \mathrm{C}$ compared with an Ogden Hyperfoam Model curve-fit for shear, uni-axial and combination cases.

\subsection{Modelling viscoelastic behaviour}

The foam modelled in this research displayed viscoelastic behavior which required modelling to simulate the foam's behaviour accurately. Stress relaxation was a prominent viscoelastic phenomenon noted during the compression and hold tests. Since bulk modulus is quite weak in this type of foamed polymer, the viscoelastic portion of the material model was dominated by the shear modulus, $G_{R}(t)$. Viscoelasticity was added to the model in the form of time based Prony-series constants based on the shear modulus of the foam, $g_{R}(t)$ :

$g_{R}(t)=1-\sum_{i=1}^{N} \bar{g}_{i}^{P}\left(1-e^{-\frac{t}{\tau_{i}^{G}}}\right)$

where $\bar{g}_{i}^{P}$ is the relaxation modulus, $\tau_{i}^{G}$ is the relaxation time. Both are material dependent properties and $\mathrm{N}$ is the order of the Prony-series.

The normalised shear modulus $g_{R}(t)$ is plotted against the $\log _{10}$ time (Figure 7 ) and ten data points (each decade of the log time plot) are extracted for use in the curve fitting procedure $\left(3^{\text {rd }}\right.$ order). More data points were then used to try and improve the curve fit, over 300 data points resulted in the model converging, with minimal error, after 8 iterations. The data is fitted using a non-linear least squares procedure to define the Prony-series parameters, $\left(\bar{g}_{i}^{P}, \tau_{i}\right)$, which are shown in Tables 4 and 5 for the respective model orders.

\begin{tabular}{|l|l|l|}
\hline $\mathbf{N}$ & $\mathbf{G ( i )}$ & Tau(sec) \\
\hline $\mathbf{1}$ & 0.0973 & 0.30639 \\
\hline $\mathbf{2}$ & 0.1740 & 11.21 \\
\hline $\mathbf{3}$ & 0.1290 & 1011 \\
\hline
\end{tabular}

Table 4: Coefficients of Prony-series model for $3^{\text {rd }}$ order case, used to model viscoelasticity 


\begin{tabular}{|l|l|l|}
\hline $\mathbf{N}$ & $\mathbf{G}(\mathbf{i})$ & Tau(sec) \\
\hline $\mathbf{1}$ & $6.17 \mathrm{E}-04$ & $1.01 \mathrm{E}-03$ \\
\hline $\mathbf{2}$ & $-1.27 \mathrm{E}-03$ & $1.89 \mathrm{E}-03$ \\
\hline $\mathbf{3}$ & $8.99 \mathrm{E}-02$ & 0.2928 \\
\hline $\mathbf{4}$ & $1.15 \mathrm{E}-01$ & 4.7441 \\
\hline $\mathbf{5}$ & $8.03 \mathrm{E}-02$ & 55.234 \\
\hline $\mathbf{6}$ & $7.72 \mathrm{E}-02$ & 629.87 \\
\hline $\mathbf{7}$ & $6.86 \mathrm{E}-02$ & 8656 \\
\hline $\mathbf{8}$ & $-3.01 \mathrm{E}-02$ & $1.74 \mathrm{E}+08$ \\
\hline
\end{tabular}

Table 5: Coefficients of Prony-series model for $8^{\text {th }}$ order case, used to model viscoelasticity

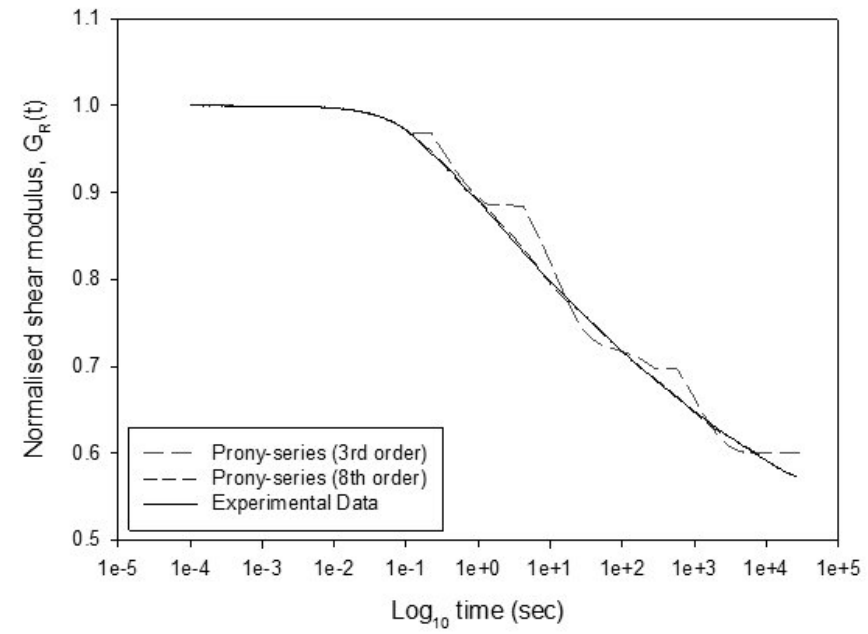

Fig. 7: Normalised shear modulus, $g_{R}(t)$ plotted against $\log _{10}$ time.

To incorporate viscoelasticity into the material model, the shear modulus of the foam, $g_{R}(t)$, is multiplied by the material constant, $\mu_{i}^{0}$, in Ogden's strain energy function (Equation 1) giving $\mu_{i}^{R}$ (Equation 7).

$\mu_{i}^{R}=\mu_{i}^{0}\left(1-\sum_{k=1}^{N} g_{k}^{-P}\left(1-e^{-\frac{t}{\tau_{k}}}\right)\right)$

The introduction of viscoelasticity using the Prony series also enables the accurate prediction of loads with variable strain rate as well as the prediction of stress relaxation at constant strain.

\section{FINITE ELEMENT SIMULATION OF STANDARD TESTS}

Standard testing procedures on polyurethane foam sample were simulated using Abaqus FE software. As previously described, Ogden's material model (Equation 1) for describing the behaviour of compressible rubber-like materials (Ogden 1972) was chosen as a suitable strain energy function. Material test data sets were fitted to the material model and material constants were extracted that gave the most accurate and robust fit available. These coefficients were examined thoroughly as their accuracy was paramount to creating accurate simulations; their stability was ensured as they passed Drucker's criterion (Simulia 2010). These modes of deformation were chosen as they were representative of the deformation undergone during seating. Only the loading curve was considered when evaluating the material parameters for the material model. Hysteretic effects were not simulated in the model presented here.

\subsection{Simulation of IFD testing}

An IFD (Indentation Force Deflection) test was conducted to demonstrate the accuracy of the material model. It can be seen from Figure 9(b) that the highest stress values were in tension along the side of the indenter. The accuracy of the model was initially validated by visually comparing material from tests and simulations at the sides of the sample and the grid deformation on the front face of the sample shown in Figure 9. The mesh used in this simulation was optimized by undertaking convergence testing. Refinement was conducted on the foam material mesh around the edge of where the indenter came into contact, as this is where mesh distortion was most likely to occur. The friction coefficient for the contact region was set to 0.75 (Mills 2000).

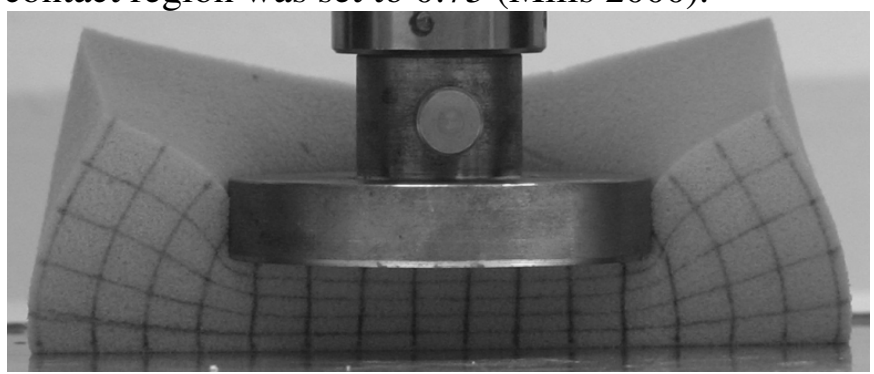

Fig.8: IFD physical test set-up, $1 / 2$ size sample compressed 50\% of initial height

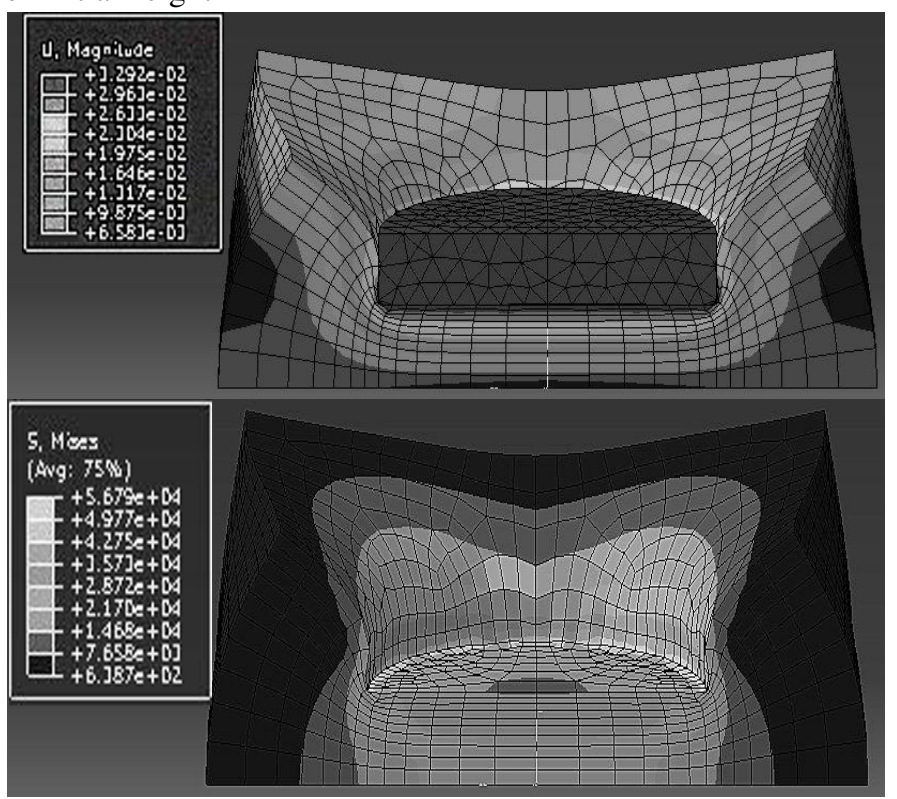

Fig.9 (a): Deformation plot of IFD test $1 \frac{1}{2}$ size simulation in Abaqus compressed by $65 \%$ of initial height (m); (b) Von Mises stress plot of IFD test $1 / 2$ size simulation in Abaqus compressed $65 \%$ of initial height $(\mathrm{Pa})$ 
The simulated force in Figure 10 was the sum of the reaction forces from the top of the indenter; this force was compared to the force from the experimental IFD test procedure. The model demonstrated good accuracy over the majority of the load curve, with some initial elastic region inconsistencies attributed to minor inaccuracies in the material model that is described by the coefficients in Table 3. This curve validated the hyperelastic section of the material model. Figure 11 indicates the accuracy of the viscoelastic model. The foam samples response to the stress relaxation procedure described in section 2.3 is demonstrated. The model predicts an instantaneous stress value which is less than that from testing. The shape of the relaxation curve closely correlates with the predicted relaxation over the extended time period of 8 hours. Hence, the Prony series model is capable of predicting the viscoelastic response of the polyurethane foam over an extended time period.

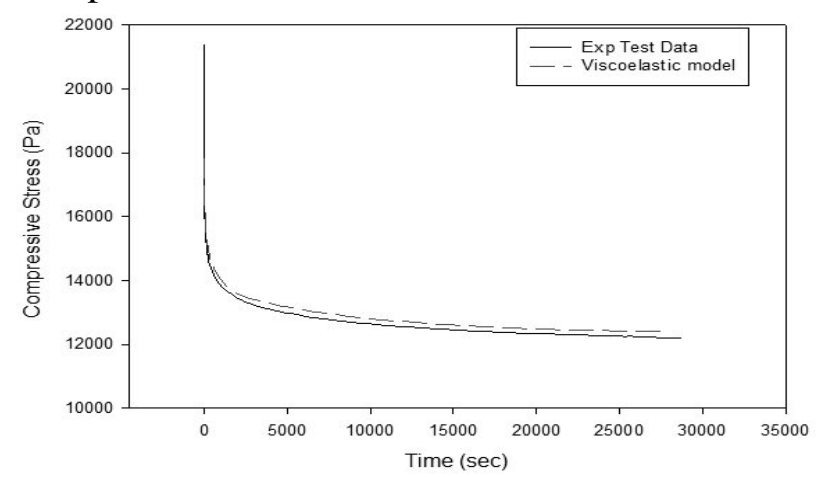

Fig.10: IFD test results compared to simulation results

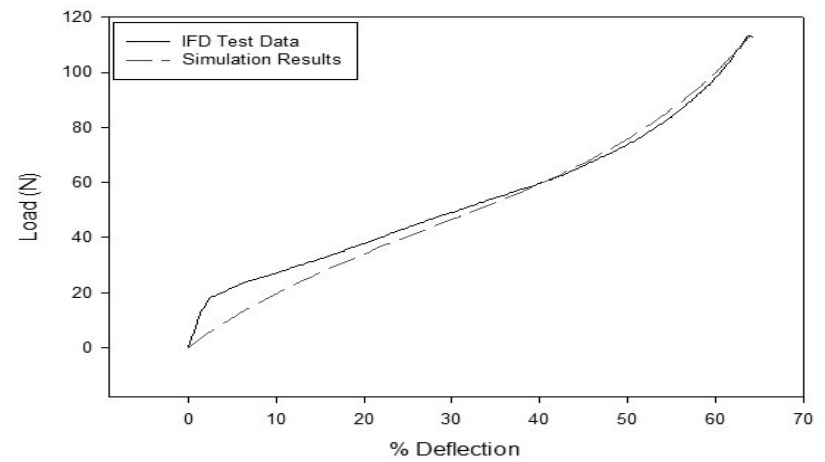

Fig.11: Graph showing accuracy of viscoelastic results over an extended time period.

\section{CONCLUSIONS}

Polyurethane foam samples were tested in uniaxial compression and simple shear modes. Test results were used to obtain suitable parameters for a second-order hyperelastic material model. This model was implemented in a simulation of an ISO indentation test. Good correlation was found between test results and simulation. The hyperelastic section of the validation process illustrated some inaccuracies in the initial strain region; this was due to similar inaccuracies within the material model. Constitutively modelling the initial elastic region for elastomeric materials is problematic and the initial region was not of in-service importance, therefore this error was not significant. The material also displayed some inherent viscoelastic properties, with stress relaxation being the most noticeable of these. Results of a compression-hold test were used to model the long term reduction of stress, which was modelled with good accuracy using a $7^{\text {th }}$ order Viscoelastic Prony Series. With both the hyperelastic and viscoelastic sections of the model validated, the user can accurately interpret displacements and stresses throughout the material during loading while also being able to monitor stress dissipation over a longer time scale.

Future work will focus on the development of this model to incorporate temperature effects, ultimately this model will provide information to aid in the prescription of wheelchair seating systems.

\section{REFERENCES}

Black, J., \& al (2007). "National Pressure Ulcer Advisory Panel's updated pressure ulcer staging system." Urol Nurs. 2(27): 144-150.

Bouten, C. V. et al. (2003). "The etiology of pressure ulcers: Skin deep or muscle bound?" Archives of Physical Medicine and Rehabilitation 84(4): 616-619.

Gibson, L. J. \& Ashby, M. F. (1997). "Cellular SolidsStructures and Properties." Second Ed.(Cambridge University Press).

Medicine, C. o. S. C. (2001). "Pressure ulcer prevention and treatment following spinal cord injury: a clinical practice guideline for health-care professionals." $\underline{\mathbf{J}}$ Spinal Cord Med. 24: S40-101.

Mills, N. (2007). Polymer Foams Handbook. Oxford, Elsevier.

Mills, N. \& Gilchrist, A (2000). "Modelling the indentation of low density Polymers." Cellular Polymers 19: 389412.

Mullins, L. (1969). "Softening of Rubber by Deformation." Rubber Chemistry and Technology 42(1): 339-362.

Ogden, R. W. (1972). "Large Deformation Isotropic Elasticity: On the Correlation of Theory and Experiment for Compressible Rubberlike Solids." Proceedings of the Royal Society of London. A. Mathematical and Physical Sciences 328(1575): 567-583.

Simulia (2010). "Analysis User's Manual." Abaqus/CAE 6.1.

Siriruk, A., Y. et al. (2009). "Polymeric foams and sandwich composites: Material properties, environmental effects, and shear-lag modeling." Composites Science and Technology 69(6): 814-820.

Voss, A. C. et al. (2005). "Long-Term Care Liability for Pressure Ulcers." Journal of the American Geriatrics Society 53(9): 1587-1592.

Wada, A. et al. (2003). "A method to measure shearing modulus of the foamed core for sandwich plates." Composite Structures 60(4): 385-390.

Ward, I. M. \& Sweeney, J. (2004). An introduction to the mechanical properties of solid polymers, Wiley. 